\title{
Lenguas indígenas y enseñanza de las matemáticas: la importancia de armonizar los términos*
}

Indigenous Languages and Teaching Mathematics: The Importance of Harmonizing Terms

Línguas indígenas e ensino das matemáticas: a importância de harmonizar os termos

\author{
Alicia Ávila-Storer** (iD orcid.org/0000-0003-0872-57xx
}

Artículo de reflexión derivado de investigación

Revista Colombiana de Educación, N.7 74. Primer semestre de 2018, Bogotá, Colombia.

Para citar este artículo: Ávila, A. (2018). Lenguas indígenas y enseñanza de las matemáticas: la importancia de armonizar los términos. Revista Colombiana de Educación, (74), 177-195.

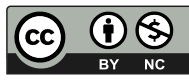

Recibido: 31/01/2017

Evaluado: 19/04/2017

* Parte de los datos en que se sustenta este artículo tienen como fuente un trabajo de investigación realizado por la autora con la participación de Silvia García, Yolanda Chávez, Carmen Gutiérrez, Leticia Iturbe y Alicia Carvajal (la ficha bibliográfica del reporte se encuentra en la lista de referencias).

** Doctora en Pedagogía por la Universidad Nacional Autónoma de México. Profesora-investigadora titular Universidad Pedagógica Nacional. Área Académica Diversidad e Interculturalidad. Ciudad de México, México. Correo electrónico: aliavi@prodigy.net.mx 


\section{Resumen}

El artículo se basa en resultados de una investigación realizada en escuelas primarias indígenas de tres regiones de México con importante presencia indígena: Chiapas, Michoacán y Puebla. Se expone la relevancia de considerar los significados que tienen los términos en las lenguas originarias y en el español cuando se enseñan las matemáticas a los niños indígenas monolingües o con distinto grado de bilingüismo. Se muestra la importancia de reconocer que los términos propios de la matemática escolar que se les enseña guardan distintas relaciones con los conceptos y términos de su cultura, a saber: (1) no siempre expresan significados idénticos a los generados en las culturas originarias; (2) estos términos algunas veces están claramente alejados en su significado; y (3) otras veces no tienen su correspondiente en esas lenguas. Se cuestiona la política de las traducciones libres o préstamos automáticos del español en la enseñanza de las matemáticas, porque con dicha estrategia se desconoce que las palabras tienen usos y significados específicos en las distintas lenguas. Se propone entonces "armonizar" los términos como un elemento que contribuya a la comprensión de las matemáticas en las aulas. Se concluye que la equidad en la enseñanza de esta disciplina a los niños indígenas empezaría por asegurarse de que comprendan lo que se dice en las clases.

\section{Palabras clave}

enseñanza de las matemáticas lenguas indígenas, educación primaria, bilingüismo, traducción de términos

\section{Keywords}

teaching mathematics; indigenous languages; elementary education; bilingualism; translating terms

\begin{abstract}
This paper is based on the results of an investigation conducted in indigenous primary schools from three Mexican regions with a significant indigenous presence: Chiapas, Michoacán, and Puebla. We discuss the importance of considering the meanings of the terms in the indigenous languages and in Spanish when teaching mathematics to indigenous children who are monolingual or with varying degrees of bilingualism. We point out the importance of recognizing that the mathematical terms they are taught in school have different meanings with regards to the terms and concepts of their culture, namely: (1) they do not always have the same meanings as those from indigenous cultures; (2) sometimes these terms have clearly distant meanings; and (3) other times they have no equivalent in those languages. We question the use of free translations or automatic loanwords from Spanish when teaching mathematics, since such practice disregards the varying uses and specific meanings of words in the different languages. We propose, then, to "harmonize" the terms as an element that contributes to the understanding of mathematics inside the classroom. We conclude that equity in teaching math to indigenous children would begin by making sure they understand what is being said in class.
\end{abstract}

\section{Resumo}

O artigo baseia-se nos resultados de uma pesquisa realizadas em escolas primárias indígenas de três regiões do México com grande presencia indígena: Chiapas, Michoacan e Puebla. Assinala-se a relevância de considerar os significados dos termos nas línguas originárias e no espanhol na aula de matemáticas para crianças indígenas monolíngues ou com diferentes níveis de bilinguismo. Evidencia-se a importância de reconhecer que os termos próprios da matemática escolar a ensinar têm diferentes relações com as concepções e termos da sua cultura, a saber: (1) nem sempre expressam significados idênticos aos gerados nas culturas originárias; (2) esses termos algumas vezes estão evidentemente afastados do seu significado; e (3) outras vezes não existe um termo equivalente nessas línguas. Questiona-se a política das traduções livres ou empréstimos automáticos do espanhol para o ensino das matemáticas, pois com essa estratégia não se leva em conta que as palavras têm usos e significados específicos nas diversas línguas. Propõe-se, então, "harmonizar" os termos como um elemento que possa contribuir para a compreensão das matemáticas nas salas de aula. Em conclusão, a equidade no ensino desta disciplina às crianças indígenas deve começar por garantir que elas possam compreender o que é dito nas aulas.

\section{Palavras-chave}

ensino das matemáticas; línguas indígenas, educação primária, bilinguismo, tradução de termos 


\section{Introducción}

Raimon Panikkar -teólogo, filósofo y científico- señaló hace tiempo que no es posible trasladar automáticamente los términos de una cultura a otra porque sus contextos de origen y uso son distintos. La palabra, según este autor, no es un vocablo objetivo e intemporal, sino temporal, comprometido, y que expresa su propio significado. Sin embargo, también dijo que es posible encontrar paralelismos en el rol que diversos conceptos tienen en dos culturas, en las funciones que cumplen en sus respectivos sistemas y se refirió a esos términos como equivalentes homemórficos (Panikkar, 1995, p. 166; Pigen, 2001, pp. 126-127).

Este artículo es mucho más modesto que una discusión filosófica o cultural de la idea introducida por Panikkar acerca de los significados -equivalentes o no- de términos aparentemente similares utilizados en diferentes culturas. Sin embargo, comienzo con tal referencia porque marca un punto de partida para entender cómo los términos propios de la matemática escolar que se enseña a los niños indígenas guardan distintas relaciones con los conceptos y términos propios de su cultura: (1) no siempre expresan significados idénticos a los generados en las culturas originarias; (2) estos términos algunas veces están claramente alejados en su significado; (3) otras veces no tienen su correspondiente en esas lenguas.

En lo que sigue, pretendo mostrar la relevancia de tomar en consideración este hecho cuando se busca hacer comprender la matemática escolar a los niños indígenas. Estos niños han crecido en una cultura que no es la mayoritaria, la cual se expresa en español. Reconozco que entre el español y el lenguaje matemático escolar también existen disonancias semánticas (o falta de armonía entre los términos). No obstante, en mi argumentación dejo al margen este problema para centrarme en las lenguas indígenas donde, como se verá, la falta de armonía entre los términos es más frecuente y aguda.

Es importante aclarar que mi objetivo no es "alinear" la enseñanza escolar con los saberes o conceptos matemáticos construidos por los niños indígenas en sus experiencias de vida. Hodge y Cobb (2016) han señalado que muchas investigaciones en educación matemática se han realizado con una orientación de alineación cultural. Desde tal perspectiva, la cultura se concibe como una forma de vida característica de una comunidad, en la cual se incluye un conjunto de prácticas relativamente estables y poco cambiantes que se heredan de padres a hijos. Los conocimientos y formas de hacer que los estudiantes desarrollan cuando participan en esas prácticas, por lo tanto, constituyen su herencia cultural. Ahora bien, el enfoque de alineación cultural identifica las discontinuidades entre las prácticas realizadas en la comunidad y las prácticas escolares como una 
fuente primaria de inequidad que deberá eliminarse. De este modo, asegurar la equidad en el aprendizaje de las matemáticas consiste en alinear las prácticas de enseñanza en el salón de clase con las prácticas no escolares en las que los estudiantes participan (Hodge y Cobb, 2016).

Mi postura es diferente. Desde una perspectiva intercultural, y ante un currículo igual para todos los niños habitantes de México, independientemente de su origen étnico y su cultura, considero que una acción obligada es establecer un lenguaje que haga comprensible a los niños lo que el profesor pretende enseñarles durante las clases de matemáticas. Esta postura probablemente es más cercana a la que Hodge y Cobb (2016) denominan Classroom orientación participation, que concibe la cultura como una red de prácticas híbridas que la gente constituye conjuntamente y que negocia en espacios específicos, tales como los salones de clase. Pero sin duda coincido con López y Küper (2000) cuando señalan que:

La dimensión intercultural de la educación está también referida tanto a la relación curricular que se establece entre los saberes, conocimientos y valores propios o apropiados por las sociedades indígenas y aquellos desconocidos y ajenos, cuanto a la búsqueda de un diálogo y de una complementariedad permanente entre la cultura tradicional y aquella de corte occidental, en aras de la satisfacción de las necesidades de la población indígena y de contribuir a la búsqueda de mejores condiciones de vida. (p. 34).

\section{El contexto'}

Antes de introducirme de lleno en el tema que anuncia el título, haré un breve rodeo con el fin de delinear el contexto en el que tiene lugar la enseñanza de las matemáticas a los niños indígenas de México, entorno de este artículo.

Según los datos que se ofrecen en el sitio de la Dirección General de Educación Indígena, hay aproximadamente 850000 niños inscritos en las escuelas primarias indígenas de México. Los profesores que enseñan en estas escuelas son más de $38000^{2}$. Al finalizar la educación primaria, los niños que asisten a este servicio educativo deberán haber aprendido -con

1 Partes importantes de este inciso, así como algunos párrafos de otros fueron publicados en la Memoria del CMO-BIRS 2015. Oaxaca. 30 de agosto-4 de septiembre de 2015. La memoria se encuentra disponible en http://prism.ucalgary.ca/handle/1880/51529.

2 Estas cifras aparecían en agosto de 2015 en el sitio web de la Dirección General de Educación Indígena de la Secretaría de Educación Pública, encargada de atender la educación de los niños indígenas de México. Hoy, que ya no existe de manera independiente esta dirección, la sep presenta cifras similares en www.sep.gob.mx/cifraseindicadores.aspx. 
algunos pocos ajustes- lo mismo que aquellos que estudian en las escuelas primarias "generales" y, además, deberán tener un manejo adecuado de la lengua originaria y del español.

El interés por el bilingüismo que aprecia las dos lenguas y que considera la segunda como un enriquecimiento y no como una sustitución (bilingüismo aditivo) es relativamente nuevo en México. Proviene de la década de 1990 y su adopción es resultado de investigaciones que constataron lo siguiente:

» La lengua materna es el principal instrumento de comunicación y desarrollo del pensamiento de una persona (Cummins, 2002; United Nations Educational, Scientific and Cultural Organization, 2003).

» No es posible desarrollar estructuras conceptuales en una lengua que se desconoce (Cummins, 2002).

» Utilizar la lengua materna genera un ambiente de confianza y seguridad en la clase y en los niños (Villavicencio, 2001; Vom Gleich, 1989, citado por Tapia, 2002).

Desde finales del siglo xx, en la educación primaria indígena que se imparte en México se ha adoptado formalmente el bilingüismo aditivo. Pero la adopción plena de este enfoque se antoja poco factible, pues está mediada por la competencia comunicativa que tienen los profesores en la lengua originaria. Veamos unos cuantos datos al respecto:

Desde hace tiempo, un requisito esencial para ser docente en las escuelas indígenas es ser hablante de una lengua originaria. Los profesores reconocen que este es el rasgo principal que ha de cubrirse para trabajar en educación indígena. Sin embargo, ellos mismos señalan que "en esto se ha fallado", pues muchos de entre ellos no cumplen el requisito.

Hace algunos años realizamos un estudio en nueve escuelas de tres estados con importante presencia indígena: Chiapas, Michoacán y Puebla ${ }^{3}$. En las escuelas visitadas se habla tzotzil, purépecha y náhuatl, respectivamente. Allí, solo el $50 \%$ de los 18 profesores participantes informaron tener alguna lengua indígena como lengua materna, el otro 50\% informó que su lengua materna es el español (Ávila et al., 2011).

Con el paso del tiempo, por lo general los profesores cuya lengua materna es el español ganan habilidad en el manejo de la lengua originaria. La aprenden con los compañeros que la conocen mejor y, principalmente, en la interacción con sus alumnos. Pero esto no ocurre siempre. También hay quienes no han aprendido la lengua, excepto algunos saludos, algunas

\footnotetext{
3 Este estudio se referenció al inicio del artículo.
} 
frases y los números hasta diez. Estos profesores pueden continuar dando clases en la educación indígena porque trabajan en zonas donde la lengua originaria ha sido casi desplazada.

Por otra parte, las actitudes hacia las lenguas originarias -incluidas las de los profesores- también determinan el uso y los fines educativos de estas en las escuelas. Por ejemplo, muchos padres quieren que la escuela sea un lugar de enseñanza del español, e incluso del inglés, pues perciben que estas son las lenguas que proporcionan oportunidades a sus hijos. Es decir que la competencia comunicativa de los docentes de educación indígena y las actitudes hacia la lengua forman una trama compleja que tiene consecuencias en las prácticas de enseñanza, incluidas las de enseñanza de las matemáticas.

\section{Las lenguas como lenguas de instrucción}

\section{Los diferentes registros de la lengua}

Según lo han puesto de relieve desde hace tiempo los lingüistas, a distintos usos de la lengua corresponden distintos grados de dominio de esta. No es lo mismo el registro conversacional que el registro académico (Cummins, 2002; López Pérez, 2007). En este último, el lenguaje es especializado y exige formas específicas de comunicación.

Ahora bien, la noción de lenguaje o registro académico generalmente ha sido utilizada para referirse al lenguaje que se usa en la educación superior, por lo que se generó también el concepto de lengua de instrucción, que permite pensar en el conjunto de los niveles educativos. Lengua de instrucción, según la definición de Mendoza Fillola, es la "lengua utilizada en la escuela como instrumento para la enseñanza-aprendizaje de los saberes" (1998, citada por López Pérez, 2007). Esta lengua, además de ser especializada, implica una forma característica de comunicación, relacionada con usos propios en cada una de las disciplinas escolares.

La lengua de instrucción sirve para expresar conocimiento acerca de los contenidos curriculares y hablar y argumentar sobre ellos, por lo que su aprendizaje posibilita el éxito escolar de los estudiantes. Pero su dominio es más difícil y tardío que el de la lengua conversacional. Además, entre los lenguajes especializados hay distintos niveles de complejidad y abstracción. Por ejemplo, para Cabré (1993, véase en López Pérez, 2007), los lenguajes como la física y la química tienen mayores niveles de especialización que otros también especializados, como el utilizado en el deporte o la peluquería. Moreno Fernández (1999), por su parte, pone en el nivel más alto de abstracción los lenguajes simbólicos, y en menor grado los científicos, los técnicos y profesionales, en ese orden. 


\section{Disciplinas escolares y lenguaje especializado}

El desigual nivel de especialización y de abstracción de los distintos lenguajes escolares, así como las consecuencias de ello, fue constatado para el caso de la lengua tzotzil, en una escuela secundaria del estado de Chiapas estudiada hace años por Benavides (2003). Ahí los usos del español y el tzotzil son distintos en cada materia. Las matemáticas se enseñan casi exclusivamente en español. Además, la inclusión del tzotzil en esta materia no tiene como finalidad explicar los temas, sino dar indicaciones de otro tipo, principalmente para la organización del trabajo. Es decir que otras materias escolares recurren a un vocabulario e ideas más próximas al contexto de los alumnos, en cambio las matemáticas se expresan en un lenguaje formal que refiere a fórmulas, clases, relaciones y propiedades abstractas alejadas de los contextos específicos, aun cuando se ejemplifican mediante casos concretos.

\section{Dificultades con la lengua en la clase de matemáticas}

Las matemáticas son vistas hoy en día como una de las áreas centrales del currículo escolar porque junto con la lengua materna ofrecen conocimientos y habilidades clave para la participación en las actividades productivas del mundo laboral y en procesos políticos y democráticos (cf. Valero, 2017). A pesar de este reconocimiento, la aplicación de encuestas y exámenes nacionales ha hecho evidente una y otra vez el déficit en el aprendizaje de las matemáticas escolares en las escuelas primarias indígenas, el cual es mucho más agudo que en el resto de las escuelas del país ${ }^{4}$. Este déficit, según interpretaciones oficiales, se debe en gran medida al poco dominio del español y, específicamente, al desconocimiento del vocabulario propio de la materia. No obstante tal reconocimiento, el problema no ha merecido una política decidida de atención específica. Por su parte, los profesores identifican: (1) problemas de traducción (de términos matemáticos incluidos en los libros de texto y otros materiales, expresados en español), y (2) falta de vocabulario en las lenguas originarias (falta de términos equivalentes a los utilizados en la matemática escolar) (Ávila et al., 2011).

4 En México es bien conocido que el desempeño en los exámenes nacionales llamados Exámenes de Calidad del Logro Educativo, conocidos como Excale, muestra las enormes desventajas de los niños indígenas en cuanto al aprovechamiento escolar previsto en el currículo. Por ejemplo, en la aplicación de 2013, el 65\% de los niños inscritos en escuelas indígenas obtuvo un nivel "por debajo del logro básico en matemáticas y español". Un dato menos conocido e igualmente elocuente es que, según cálculos del Instituto Nacional para la Evaluación de la Educación (inee), en el medio indígena el 31 \% de los niños inscritos en sexto grado en el ciclo 2013-2014 había reprobado al menos un año escolar. 
Los profesores han tratado de resolver estas dificultades por dos vías: generando términos no existentes en la lengua originaria (neologismos), o mediante préstamos del español. Esto se ha hecho sobre todo mediante iniciativas individuales: cada maestro o grupo de maestros toma decisiones y realiza acciones para resolver este aspecto de la enseñanza en su salón de clases o en su escuela. Ocasionalmente los esfuerzos alcanzan el nivel institucional, por ejemplo mediante cursos donde se ayuda a los docentes a la producción y traducción de términos ${ }^{5}$. Pero como se verá, al tratarse de dos culturas, el tema es mucho más complejo que la simple creación libre o el préstamo de términos.

\section{La dificultad para generar términos o hacer com- prensibles términos no armonizados. Tres ejemplos}

\section{Ejemplo 1. Las figuras geométricas}

En términos generales, el currículo de matemáticas que guía la enseñanza y el aprendizaje de esta materia en las primarias indígenas es el mismo que orienta la enseñanza que se imparte a todos los niños mexicanos. De este modo, los niños indígenas deben aprender las figuras geométricas tradicionalmente incluidas en el currículo, las operaciones aritméticas básicas y sus correspondientes algoritmos, la proporcionalidad o el plano cartesiano, apoyados en los mismos materiales que se proporcionan a los demás niños del país.

El círculo, el cuadrado, el rectángulo y el triángulo son figuras que se incluyen en todos los grados de la educación primaria mexicana desde hace muchas décadas ${ }^{6}$. No obstante, aunque sin analizarlas ni visualizar todas sus propiedades, es muy probable que los niños que asisten a escuelas no indígenas identifiquen las figuras desde el primero o segundo grado. También es probable que sepan sus nombres y puedan reproducirlas con relativa corrección. Esto porque las figuras, debido a su uso social frecuente, son familiares a los niños desde sus primeros años. Pero, ¿qué ocurre en los contextos donde se habla náhuatl, purépecha o tzoztil? Inicio la respuesta por este último.

5 Por ejemplo, durante la indagación que da sustento parcial a este escrito, en el estado de Michoacán se había impartido un curso para sugerir a los docentes términos útiles para la enseñanza y aprendizaje de la aritmética; se incluyeron términos relativos a las operaciones aritméticas, a sus partes y a sus resultados.

6 El cuadrado también es un rectángulo (tiene cuatro ángulos rectos). Sin embargo, conforme a la tradición que prevalece en las escuelas primarias mexicanas, se habla de una u otra figura sin considerar las clases o subclases a las que pertenecen, más allá de ser cuadriláteros. En lo que sigue me referiré a ellas de dicha manera, pues así las refieren también los profesores con quienes conversamos. 


\section{El tzotzil}

Los términos rectángulo o triángulo -según los datos que recabamos- no se utilizan en tzotzil, lengua en las que solo se usan términos cercanos a cuadrado y círculo. Sin embargo, los significados de estos dos términos no son similares a los que se les da en español y en el currículo escolar. Círculo se refiere más a un "encierro" que a una figura de ciertas propiedades, sin importar la forma. El marco de una fotografía o el contorno de un corral pueden ser "un encierro". La idea de cuadrado -según nos dice nuestra traductora- refiere a una idea de la Tierra que en esa cultura se concibe con cuatro lados iguales y cuatro "puntos de encuentro"; el término cuadrado, pues, tampoco refiere a una figura con ciertas propiedades y que constituye un contenido curricular.

\section{El náhuatl}

Algo similar nos dice nuestra traductora hablante del náhuatl de la Sierra Norte de Puebla, punto geográfico de referencia de este escrito:

En esta lengua, la palabra rectángulo no existe. A veces se dice que "algo está alargado", como por ejemplo una casa o un terreno. La palabra utilizada para expresar esta idea es tlatshuéyac.

Círculo como tal tampoco existe. Lo que se usa en esta lengua es una palabra para hablar de "las cosas redondas": una pelota, un cántaro, una niña regordeta... La palabra para expresar esta idea es yahualtik.

Aquí nuestra entrevistada dice, además, que la noción de cuadrado no se usa, sino la que ella considera rombo y que vincula a los bordados (véase la figura 1):



Figura 1. Copia del dibujo de la traductora realizado para explicar la utilidad del "rombo" en la elaboración de bordados

7 Las referencias a las lenguas originarias en esta parte del escrito se sustentan en entrevistas a docentes realizadas durante la investigación mencionada al inicio del artículo, y en entrevistas Ilevadas a cabo en 2016 a Cecilia Díaz, Yéssica Hernández y Puki Lucas Hernández, a quienes agradezco sinceramente su buena disposición para compartir su conocimiento y experiencia con su idioma materno. 
Yéssica: [Esta figura es útil] Porque si está así (se refiere a que está descansando sobre uno de sus vértices y una de sus diagonales está en posición vertical), sí cabe la figura; aquí le ponen la colita del gallo (señala el vértice izquierdo, garabatea sobre el cuadrado); aquí le ponen la cabecita (señala el vértice superior). Si es cuadrado, no cabría, así cabe mejor la figura (Yéssica, hablante del náhuatl).

\section{El purépecha}

En purépecha sí existe el término círculo -wirhipu-. Según nuestro traductor, la palabra refiere a algo redondo; su raíz verbal whiri, tiene el significado "ser redondo", o "rodar", y el sufijo pu es un nominalizador. Se utiliza cuando las personas hacen un círculo humano, cuando trazan un círculo en el suelo, o cuando hacen alguna figurita de cualquier material. También se utiliza para referirse a la forma del sol, o cuando hay luna llena.

En cambio, en la lengua purépecha no existe un término equivalente a cuadrado, porque no existe el concepto cuadrado, aunque hay una manera de indicar este sentido, que es "cuatro esquinas": t'amu tsuminti jukari "algo que tiene cuatro esquinas", que se usa para referirse a todo lo que tenga cuatro esquinas y que [los lados] sean de la misma proporción.

El término rectángulo tampoco existe, pero sí hay una forma de expresar algo similar: "algo que tiene cuatro esquinas y es alargado": T'amu tsuminti jukari ka yókurhini. Esta expresión se usa para referirse a todo lo que tiene cuatro equinas y unas partes son más alargadas que otras.

La palabra triángulo, en el sentido que se utiliza en la escuela, no existe en ninguna de las lenguas comentadas. En purépecha, de nuevo, se usa una expresión para referirse a todo lo que tenga tres esquinas y lados la misma proporción: Taminu tsuminti jukari. Este vocablo, considerando su relación con el currículo escolar, correspondería al triángulo, pero solo al equilátero, ya que sus lados han de ser de igual tamaño.

Como se ve, no todos los términos con los que nombramos las figuras geométricas existen en las lenguas originarias a las que nos hemos referido, o estos términos no tienen el mismo significado que se les asigna en la matemática que se enseña en las escuelas. Con ello se constata que

» El sentido de las palabras se elabora en el uso social y dentro de una cultura. Los términos (y los conceptos a los que nombran) en las culturas que hemos referido no tienen un significado equivalente al que tienen en el currículo escolar; por ejemplo, la idea de cuadrado como la forma de la Tierra, con cuatro puntos de encuentro, se distancia claramente de la idea escolar de cuadrado, en la que el interés se centra en las propiedades de dicha figura y su análisis. 
» Los términos para evocar las formas están vinculados a referentes específicos, no constituyen en sí conceptos abstractos, idealizaciones, como se pretende con los términos correspondientes en el currículo escolar (en este se busca que el término haga referencia a clases de figuras que cumplen ciertas propiedades).

En síntesis, no todos los significados sobre las figuras geométricas que se han construido en las culturas referidas en este escrito están "armonizados" en el uso social y modos de nombrar en el español y en el lenguaje escolar.

\section{Ejemplo 2. La multiplicación}

En seguida expongo un caso en el cual el significado de los términos, sin ser diferente, no es tan fácil de captar debido a las escasas experiencias que los implican. Este caso lo introdujo un profesor con apenas dos años de servicio y que trabajaba en una aislada comunidad de Los Altos de Chiapas:

Yo encontraba esa dificultad... porque multiplicación, ¿qué es multiplicación?, me dijeron [los alumnos] pues, así rapidito me agarraron... [desprevenido]... "multiplicación"... y no, [no supe]... Después investigué cómo se podía decir multiplicación... podría ser duplicar tal cantidad que te marca aquí (se refiere a uno de los factores de la multiplicación), según lo que te pida el otro (el otro factor). Y, así traduciendo a mi lengua, así, ya es más fácil. Sí, pero que te diga así multiplicación, rápido, como que pues... ¿multiplicación como se dice en mi lengua?, pues no, no hay traducción [...]

Y división... ahí no tuve tanto problema, porque división, ellos [los alumnos] habían manejado siempre ese término, yo creo que es porque... dividen su terrenitos: qué tanto le meten de rábano, qué tanto le meten de flores, ¿isí?, siempre usan división. (Profesor Raúl; Los Altos de Chiapas)

Nuestras entrevistadas nos proporcionan claridad sobre el punto:

"Es cierto, dividir sí existe, porque sí se reparte, así sí se hace, pero multiplicar, no tanto, porque la idea es más como de la cosmovisión occidental, porque es con la idea de acumular cosas, y para nosotros no existe esa idea de ir acumulando cosas, porque en nuestra cosmovisión no tenemos la idea de adquirir más de lo que la tierra nos da, no acumulamos riquezas, y es lo que tenemos, pero no haces cosas para tener más y más. División sí existe la idea, porque ahí sí piensas por ejemplo en cómo repartes para que lo que tienes te alcance para toda la familia". (Cecilia, hablante de tzotzil)

"En lo personal, creo que sí [se tiene la idea de dividir], porque a diario ven lo de repartir. Tal vez tenga razón el maestro, porque como las familias en la comunidad son muy extensas, reparten la comida, la 
dividen entre todos, eso es común, y multiplicar... es más difícil de ejemplificar... por ejemplo puede ser cuando van a cortar café, va toda la familia, y por ejemplo tu hermanito cortó tres, tu mamá 30, juntan con lo que cortó el papá, y la mamá, y el hermanito, porque toda la familia va al corte [de café] ... (repentinamente guarda silencio, piensa algunos segundos, luego continúa)... pero la situación que le comento es más bien de sumar que de multiplicar [¿verdad?] (se sonríe)". (Yéssica, hablante de náhuatl)

Se ve de nuevo cómo la actividad comunitaria y la cultura del lugar son generadoras (o no) de significados que facilitan el aprendizaje de los conceptos y términos matemáticos escolares. Reconozco que las comunidades son abiertas y por ello mismo cambiantes. Reconozco también que los profesores pueden imaginar y proponer situaciones donde la multiplicación se concretice con pertinencia cultural. Empero, eso no invalida el hecho de que haya ideas que circulan en las comunidades y ofrecen a los niños referentes que son armónicos con lo que aprenden en la escuela, mientras que otras no.

\section{Ejemplo 3. El plano cartesiano y la ubicación en el espacio}

Por último haré referencia a un tema que, con variantes, traté en otro escrito: el plano cartesiano (Ávila, 2016). Este es un tema incluido desde 1972 en el currículo de educación primaria en México. El caso es interesante y útil para nuestro análisis puesto que hemos sabido de la preocupación de un connotado sociolingüista por generar los términos correspondientes en lengua purépecha. Este investigador trabajó con un grupo de profesores con el objetivo de generar los términos escolares necesarios para tratar el plano y nos informa de una larga discusión al respecto, que dio por resultado el siguiente acuerdo:

... Ilamar al plano [cartesiano] mismo kuirunharhitakata, "trazar donde -el lugar- la superficie del papel". El eje X Ileva el nombre xanharaku 'camina horizontalmente' (imperativo), mientras que el eje $Y$ se llama tirhipani tihirharhitaku, literalmente, "colgar algo verticalmente". Buscan nombres para las distintas operaciones y desarrollan esa misma tarde una clase para el próximo día donde usarán los conceptos y validarán su funcionamiento". (Hamel, 2007, pp. 177-178).

Para ponderar el alcance y los límites de esta forma de trabajo con la lengua, valen las preguntas siguientes: ¿En la comunidad de referencia se usa el sistema cartesiano como sistema de ubicación en el espacio?, o ¿existen y se utilizan otros sistemas de localización? De ser así, ¿cuál es la lógica que los sustenta? En el terreno de la enseñanza: ¿tiene suficiente sentido para los niños hablar sobre el plano cartesiano y, además, referirse a él con base en los términos generados: algo que se escribe en el papel, 
caminar horizontalmente y colgar algo verticalmente? ¿Los términos utilizados evocarán en los niños los referentes de significado pertinentes para el aprendizaje del tema?

Creo válido tener dudas sobre lo anterior porque es bien sabido que los antropólogos hablan de al menos dos sistemas de localización utilizados por los pueblos originarios: (1) el egocéntrico, que toma como base al propio individuo; y (2) el geocéntrico, que toma algún elemento geográfico, como el sol, la montaña, la parte más baja de la localidad, etc. como referente para establecer la ubicación de las personas y los objetos (Hernández, 2008; León, 1993, citado por Benavides, 2003).

De acuerdo con la explicación que nos dio nuestro intérprete en lengua purépecha, el sistema de localización utiliza los puntos cardinales, pero las orientaciones espaciales específicas generalmente se elaboran indicando lo que hay en el pueblo o alrededor de él, como pueden ser: cerros, lagos, ríos, casas, canchas, árboles grandes, escuelas, o algún otro referente, por ejemplo: Xanara jurhimpiti ka jimanka ta k'eri jaka jima kaniku para jini kópekuarhu ísi nirani "Camina derecho y ahí donde está la casa grande da vuelta yendo hacia la plaza".

En el caso de nuestro estudio con niños, identificamos un sistema de ubicación en el espacio que no coincide con el europeo, el cual tiene precisamente el plano cartesiano como eje del sistema de localización y que es el que se incluye en los libros de texto y los programas oficiales. El sistema utilizado por los niños de nuestra indagación es predominantemente geocéntrico, y se vale del señalamiento de puntos específicos como árboles, casas y otros referentes geográficos para orientarse, por ejemplo: "En la escuela, allá derecho, allá hay un poste y vas pa'bajo, derecho y, ahí está la placita y te das una vuelta y ahí está la iglesia" (Gabriel, zona lacustre de Pátzcuaro).

En este contexto de localización, nos llamó poderosamente la atención que, salvo algunas excepciones, los niños que participaron en nuestra investigación no utilizaban las palabras derecha e izquierda al describir los trayectos para trasladarse de un lugar a otro de su comunidad o cerca de ella.

Los factores intervinientes en esto sin duda pueden ser muchos: las comunidades visitadas son pequeñas, las casas no están alineadas, muchas veces están dispersas y se llega de un lugar a otro sin necesidad de ser tan precisos ni utilizar sistemas de coordenadas como sí se requiere en las ciudades, organizadas en forma de cuadrícula, por calles y cuadras.

Ya nuestro colega purépecha nos informó sobre cuáles son los referentes para la localización en comunidades pequeñas que hablan esa lengua. Nuestra intérprete del tzotzil, con respecto a la pregunta, expresa: 
... Tiene mucho que ver con el contexto, porque por ejemplo, yo a éste le entiendo (señala un plano simplificado del centro de un pueblo que aparece en el libro de texto oficial de matemáticas de segundo grado); y un niño de mi comunidad le entiende porque el pueblo ya está así, por cuadras y calles, pero si vamos a otro pueblo que no tiene ni cuadras, ni calles, ni esquinas, que sólo son veredas, puede ser que no le va a entender... porque no lo vive [...]. En cambio, si le das un mapa, le dices "Explícame cómo vas de tu casa a la escuela, o a la iglesia", pero sin calles, entonces sí lo va a entender, [y te diría]: En vez de que "Primero paso una esquina y doy vuelta [a la izquierda] en el mercado", "Primero, paso un árbol de aguacate, luego paso el potrero, luego [...] y sí entendería que eso es un croquis". (Cecilia, hablante del tzotzil).

Lo que esta joven está haciendo es señalar en sus propias palabras que son los referentes de quien interpreta, los significados que ha construido con el uso, los que favorecen o dificultan la comprensión. Si esos referentes (que son producto de la experiencia y que dotan de significado a las palabras) no existen, la comprensión se torna mucho más difícil.

Quien nos tradujo del náhuatl al español responde de manera similar:

Nosotros [en náhuatl] decimos arriba y abajo, si voy para este lado (se coloca en una posición seguramente conveniente, y levanta su brazo derecho), es que para nosotros la derecha siempre es norte, va siempre hacia arriba, y la izquierda (levanta el brazo izquierdo) se supone que baja. [...] Si [llegando a la carretera] se fueran para acá (hacia la izquierda), dirían "vamos a bajar", vamos a tomar la urbana y vamos a bajar al pueblo, y para acá (hacia la derecha) dirían "vamos a subir". (Yéssica, hablante de náhuatl).

Nuestra traductora del tzotzil de nuevo nos dice algo con un sentido muy similar:

Es que como es de ida, porque si esta es mi casa (señala la representación de una casa cercana a la escuela en un croquis), es que me voy para el pueblo, salgo de la casa y llego a la carretera, pero no puedo decirles izquierda o derecha [porque no existen las palabras], entonces, para decirles a qué lado voy, digo batsï kom, que es como mi mano verdadera, [y que puede entenderse como "derecha"], y si tengo que irme de este lado [hace un ademán con la mano izquierda], entonces digo tsét k’om que es como mi mano falsa. (Cecilia, hablante del tzotzil).

También se nos dijo que los términos derecha e izquierda (o los equivalentes en la lengua), generalmente no son necesarios en lo cotidiano para expresar la ubicación o la orientación, por lo que tienen muy poco 
uso. Los niños (y en general las personas) para describir un trayecto más bien recurren a referentes concretos: los transportes y las señalizaciones que llevan; como las combis ${ }^{8}$ y el letrero que indica hacia dónde van:

Pues es que la combi tal vez es un referente para ellos, saben que la combi de este lado [de la carretera] va para cierto lugar, y que la del otro lado, va para otro lugar. Ya saben a dónde los va a llevar [se guían con el letrero], la combi es el referente más cercano para ellos. Ya saben que si dices lo que dice el letrero, de seguro te va a llevar adonde quieres ir. (Yéssica, hablante del náhuatl).

El plano cartesiano, y las palabras derecha o izquierda, a quienes vivimos en las ciudades nos son tan familiares que ni reparamos en su uso. En cambio, como hemos visto, son ideas y términos ajenos a los niños habitantes de comunidades pequeñas, como en las que viven la gran mayoría de los niños indígenas. Al usarlas en las clases de matemáticas de una manera transparente, como si estuviesen armonizadas con los referentes de los niños, es probable que estas ideas y estas palabras obstaculicen la comprensión de lo escolar que se quiere comunicar.

Aunque ya no lo documento con detalle para no fatigar en exceso al lector, también encontré que las frases "arriba" o "abajo", como recursos de localización, tienen sentidos propios y diversos en las distintas comunidades: ¿se trata del norte y el sur?, ¿o se trata del este y el oeste? Para contestar, habrá que saber cuál es la comunidad de referencia, pues Hernández (2008) documentó esta forma de referirse al norte y al sur en Gardenias, una comunidad de la Sierra Norte de Puebla donde se habla el náhuatl. En cambio, De León (1994, citado por Benavides, 2003) menciona que en Zinacantan, Chiapas, "arriba" y "abajo" corresponden, respectivamente, al este $y$ al oeste.

Ahora bien, es importante señalar que muy probablemente no con todos los conceptos o procedimientos matemáticos se dé la falta de armonización que aquí he destacado. Pero esto es solo una hipótesis, pues el contexto de uso de los términos en lenguas originarias y su nivel de "armonía" con el español es tema de investigación pendiente.

\section{Una respuesta docente a las dificultades con los términos}

La estrategia que algunos maestros han desarrollado para enfrentar didácticamente la complejidad de la traducción es utilizar el diccionario (escolar) del español, porque, dicen, "aquí se ve lo que significa un término".

8 Las combis son pequeñas camionetas de transporte público colectivo disponibles hasta en las zonas más apartadas; en ellas caben entre seis y ocho personas. 
Lo anotado en el diccionario -aun sin ser éste de matemáticas- seguramente resulta más ilustrativo que sus intentos personales por explicar los conceptos en juego. Muchos profesores siguen esta estrategia, aunque al hacerlo introducen en sus clases las limitaciones que aquí hemos señalado.

No obstante, esperar que los profesores realicen esta tarea es transferirles una responsabilidad que los rebasa. Una profesora cuyos alumnos son hablantes del náhuatl lo dijo así:

Para un maestro, no es sencillo hacer la traducción; para traducir, hay que saber muy bien la lengua y, además, no siempre existen los referentes suficientes para hacer esa traducción [...], porque vienen muchas palabras que sí, no se pueden traducir, se nos dificulta porque no se pueden traducir bien al náhuatl. [Al ejemplificar, la profesora cita términos como ángulo].

Lo anterior llama la atención sobre la importancia de que en la formación de profesores para las escuelas indígenas se incluya el aprendizaje o perfeccionamiento de la lengua en la que el docente desarrollará su labor. También muestra la importancia de que se desarrollen lenguajes específicos para las distintas materias. Este desarrollo, para ser pertinente, deberá sustentarse en un conocimiento del significado de las palabras en la lengua originaria y en la creación de zonas de significados comunes entre dichas lenguas y el español.

\section{Conclusiones}

» No siempre los significados asignados a los conceptos matemáticos que se enseñan en la escuela son los mismos que les asignan los niños que han crecido inmersos en una cultura indígena y que han sido socializados en la lengua de su comunidad.

» Uno de los principales retos que enfrenta la enseñanza de las matemáticas en la educación indígena es el manejo de la lengua originaria y su relación con el español, lengua en la que hasta hoy ha sido pensado y elaborado el currículo escolar para los niños indígenas.

» La postura tradicional considera que el problema de enseñanza y aprendizaje de las matemáticas en el medio indígena se reduce a generar (libremente) los términos necesarios para poder hablar de los contenidos incluidos en el currículo, o a pedirlos prestados al español.

» Como aquí se ha visto, y desde una perspectiva intercultural, la tarea debe ir más allá porque se hace necesario armonizar los términos. Se han de identificar los usos sociales y los significados que 
los términos tienen en una y otra lengua para determinar si, como dijera Panikkar, son "equivalentes homeomórficos" o si su disonancia semántica obliga a esfuerzos didáctico-lingüísticos más amplios para crear zonas de significado común que favorezcan la comprensión de los conceptos que los términos nombran.

» Para realizar la tarea de armonizar los términos, sería muy útil generar en las aulas espacios de habla, de comunicación y de intercambio entre profesores y alumnos, de manera que los niños también colaboraran en la construcción de un lenguaje matemático escolar pertinente y significativo. La participación de padres de familia y de especialistas que miren desde distintas perspectivas el asunto, también sería esencial.

» La construcción de un currículo realmente intercultural en matemáticas que, en palabra de López y Küper (2000), busque un diálogo y una complementariedad permanente entre la cultura tradicional y aquella de corte occidental, es una tarea pendiente en México. Confío en que estudios y reflexiones como los que aquí expuse contribuyan, así sea modestamente, a dicha construcción.

\section{Reflexión adicional}

No quiero dejar la sensación de una postura que limita los problemas de la enseñanza de las matemáticas a la lengua. Las limitaciones en los procesos de enseñanza y aprendizaje de las matemáticas en las escuelas indígenas no es solo un problema de lengua: es un problema de didáctica y de condiciones en las que se desarrolla la enseñanza; pero como aquí hemos visto, sí está vinculado con la lengua.

\section{Referencias}

Ávila, A. (directora). García, S.; Chávez, Y.; Gutiérrez, C.; Iturbe, L. y Carvajal, A. (2011). Educación matemática en escuelas indígenas, análisis de sus condiciones y problemas. Reporte inédito. México. Universidad Pedagógica Nacional/Dirección General de Educación Indígena.

Ávila, A. (2015). Enseñanza de las matemáticas y lenguas originarias en escuelas indígenas de México. Primeras notas sobre un vínculo complejo. Memorias del смо-вirs 2015: Concept Study - Profound Understanding of Teachers' Mathematics. 30 de agosto - 4 de septiembre de 2015. Recuperado de http://prism.ucalgary.ca/handle/ 1880/51529. 
Benavides, G: (2003). Alternancia y conflicto lingüístico en un espacio escolar de Los Altos de Chiapas (tesis de maestría). Centro de Investigaciones y Estudios Superiores en Antropología Social (Ciesas), México.

Cabré, M. T. (1993). Terminología. Teoría, metodología, aplicaciones. Barcelona: Antártida/Empúries.

Cummins, J. (2002). Lenguaje, poder y pedagogía. España: Ministerio de Educación y Cultura/Morata.

De León, L. (1994). La llegada del alma: lenguaje e infancia en Zinacantán. México: Centro de Investigaciones y Estudios Superiores en Antropología Social (Ciesas).

Hammel, E.; Brumm, L.; Carrillo, A.; Loncón, E.; Nieto, R. y Silva, E. (2004). ¿Qué hacemos con la castilla? La enseñanza del español como segunda lengua en un currículo intercultural bilingüe de educación indígena. Revista Mexicana de Investigación Educativa, 9(20), 83-107.

Hernández, J. I. (2008). Marcos de referencia y categorías de espacialidad en el náwat de Gardenias, Hueyapan, Puebla (tesis de maestría). Centro de Investigaciones y Estudios Superiores en Antropología Social (Ciesas), México.

Hodge, L. y P. Cobb (2016). Two views of culture and their implications for mathematics teaching and learning. Urban education. First in line (pp. 1-25). Recuperado de www.journalssage.pub.com.

Instituto Nacional para la Evaluación de la Educación (INEE). 2014). La población indígena en México. Recuperado de www.inee.gob.mx.

López, L. E. y Küper, W. (2000). La educación intercultural bilingüe en América Latina: balance y perspectivas. [Documento de trabajo]. Cochabamba y Lima, Perú: Cooperación Técnica Alemana (GTz).

López Pérez, V. (2007). La lengua de instrucción (ı): aproximación teórica y perspectiva de análisis en el marco de la enseñanza del español (L2). Revista Española de Lingüística Aplicada, 20, 85-107.

México, Secretaría de Educación Pública. (2015-2016). Principales cifras del sistema educativo nacional. Recuperado de www.planeacion. sep.gob/estadisticaeindicadores.aspx.

Panikkar, R. (1995). Invisible harmony. Essays on contemplation and responsibility. Mineápolis: Fortes Press.

Pigen, J. (2001). Interculturalidad, pluralismo radical y armonía invisible. Ilú. Revista de Ciencias de las Religiones. Samadhanam, 117-131.

Tapia Fernández, Y. (2002). Estrategias metodológicas de enseñanza y uso de lenguas en docentes de escuelas EBı en el distrito de Mañazo-Puno (tesis de maestría). Universidad Mayor de San Simón, Cochabamba, Bolivia.

United Nations Educational, Scientific and Cultural Organization, UNESCO. (2003). Education in a multilingual world. París: Autor. Recuperado de www.unesco.org/education. 
Valero, P. (2017). El deseo de acceso y equidad en la educación matemática. Revista Colombiana de Educación, (73), 99-128.

Villavicencio, M. (2001). El aprendizaje de las matemáticas en el Proyecto Experimental de Educación Bilingüe de Puno y en el Proyecto de Educación Intercultural de Ecuador: reflexiones sobre la práctica y experiencias relacionada. En A. Lizarzaburu y G. Zapata (comps.) Pluriculturalidad y aprendizaje de la matemática en América Latina (pp. 167-190). Madrid: PREIB Andes/ DSE/ Morata. 